\title{
Inequalities and boundedness for commutators related to integral operator with general kernel
}

Jiasheng Zeng*

\section{"Correspondence:}

zengjiashenga@163.com College of Mathematics, Hunan University of Commerce, Changsha, 410205, P.R. China

\begin{abstract}
In this paper, we establish the sharp maximal function inequalities for the commutators related to some integral operator with general kernel and the $B M O$ and Lipschitz functions. As an application, we obtain the boundedness of the commutators on Lebesgue, Morrey and Triebel-Lizorkin space. The operator includes Littlewood-Paley operators, Marcinkiewicz operators and Bochner-Riesz operator. MSC: 42B20; 42B25

Keywords: commutator; Littlewood-Paley operator; Marcinkiewicz operator; Bochner-Riesz operator; sharp maximal function; Morrey space; Triebel-Lizorkin space; $B M O$; Lipschitz function
\end{abstract}

\section{Introduction and preliminaries}

As the development of singular integral operators (see [1-3]), their commutators have been well studied (see [4-6]). In [5-7], the authors prove that the commutators generated by the singular integral operators and $B M O$ functions are bounded on $L^{p}\left(R^{n}\right)$ for $1<p<\infty$. Chanillo (see [8]) proves a similar result when singular integral operators are replaced by the fractional integral operators. In [9-11], the boundedness for the commutators generated by the singular integral operators and Lipschitz functions on Triebel-Lizorkin and $L^{p}\left(R^{n}\right)(1<p<\infty)$ spaces are obtained. In [12], some singular integral operators with general kernel are introduced, and the boundedness for the operators and their commutators generated by $B M O$ and Lipschitz functions are obtained (see $[12,13])$. The purpose of this paper is to prove the sharp maximal function inequalities for the commutator associated with some integral operator with general kernel and the $B M O$ and Lipschitz functions. As an application, we obtain the boundedness of the commutator on Lebesgue, Morrey and Triebel-Lizorkin space. The operator includes Littlewood-Paley operators, Marcinkiewicz operators and Bochner-Riesz operator.

First, let us introduce some notations. Throughout this paper, $Q$ will denote a cube of $R^{n}$ with sides parallel to the axes. For any locally integrable function $f$, the sharp maximal function of $f$ is defined by

$$
M^{\#}(f)(x)=\sup _{Q \ni x} \frac{1}{|Q|} \int_{Q}\left|f(y)-f_{Q}\right| d y ;
$$

○2014 Zeng; licensee Springer. This is an Open Access article distributed under the terms of the Creative Commons Attribution License (http://creativecommons.org/licenses/by/2.0), which permits unrestricted use, distribution, and reproduction in any medium, provided the original work is properly cited. 
here, and in what follows, $f_{Q}=|Q|^{-1} \int_{Q} f(x) d x$. It is well known that (see $\left.[1,2]\right)$

$$
M^{\#}(f)(x) \approx \sup _{Q \ni x} \inf _{c \in C} \frac{1}{|Q|} \int_{Q}|f(y)-c| d y .
$$

We say that $f$ belongs to $B M O\left(R^{n}\right)$ if $M^{\#}(f)$ belongs to $L^{\infty}\left(R^{n}\right)$ and define $\|f\|_{B M O}=$ $\left\|M^{\#}(f)\right\|_{L^{\infty}}$. It has been known that (see [14])

$$
\left\|f-f_{2^{k} Q}\right\|_{B M O} \leq C k\|f\|_{B M O} .
$$

Let

$$
M(f)(x)=\sup _{Q \ni x} \frac{1}{|Q|} \int_{Q}|f(y)| d y .
$$

For $\eta>0$, let $M_{\eta}(f)(x)=M\left(|f|^{\eta}\right)^{1 / \eta}(x)$.

For $0<\eta<1$ and $1 \leq r<\infty$, set

$$
M_{\eta, r}(f)(x)=\sup _{Q \ni x}\left(\frac{1}{|Q|^{1-r \eta / n}} \int_{Q}|f(y)|^{r} d y\right)^{1 / r} .
$$

The $A_{p}$ weight is defined by (see [1])

$$
\begin{aligned}
A_{p}= & \left\{w \in L_{\mathrm{loc}}^{1}\left(R^{n}\right): \sup _{Q}\left(\frac{1}{|Q|} \int_{Q} w(x) d x\right)\left(\frac{1}{|Q|} \int_{Q} w(x)^{-1 /(p-1)} d x\right)^{p-1}<\infty\right\}, \\
& 1<p<\infty,
\end{aligned}
$$

and

$$
A_{1}=\left\{w \in L_{\mathrm{loc}}^{p}\left(R^{n}\right): M(w)(x) \leq C w(x), \text { a.e. }\right\} .
$$

For $\beta>0$ and $p>1$, let $\dot{F}_{p}^{\beta, \infty}\left(R^{n}\right)$ be the weighted homogeneous Triebel-Lizorkin space (see $[11])$.

For $\beta>0$, the Lipschitz space $\operatorname{Lip}_{\beta}\left(R^{n}\right)$ is the space of functions $f$ such that

$$
\|f\|_{\operatorname{Lip}_{\beta}}=\sup _{\substack{x, y \in R^{n} \\ x \neq y}} \frac{|f(x)-f(y)|}{|x-y|^{\beta}}<\infty .
$$

In this paper, we will study some integral operators as follows (see [12]).

Definition 1 Let $F_{t}(x, y)$ be defined on $R^{n} \times R^{n} \times[0,+\infty)$ and $b$ be a locally integrable function on $R^{n}$, set

$$
F_{t}(f)(x)=\int_{R^{n}} F_{t}(x, y) f(y) d y
$$

and

$$
F_{t}^{b}(f)(x)=\int_{R^{n}}(b(x)-b(y)) F_{t}(x, y) f(y) d y
$$

for every bounded and compactly supported function $f$. 
Let $H$ be the Banach space $H=\{h:\|h\|<\infty\}$. For each fixed $x \in R^{n}$, we view $F_{t}(f)(x)$ and $F_{t}^{b}(f)(x)$ as the mappings from $[0,+\infty)$ to $H$. Set

$$
T(f)(x)=\left\|F_{t}(f)(x)\right\|
$$

which $T$ is bounded on $L^{2}\left(R^{n}\right)$. The commutator related to $F_{t}^{b}$ is defined by

$$
T^{b}(f)(x)=\left\|F_{t}^{b}(f)(x)\right\|
$$

and for $F_{t}$ we find that there is a sequence of positive constant numbers $\left\{C_{k}\right\}$ such that for any $k \geq 1$,

$$
\int_{2|y-z|<|x-y|}\left(\left\|F_{t}(x, y)-F_{t}(x, z)\right\|+\left\|F_{t}(y, x)-F_{t}(z, x)\right\|\right) d x \leq C
$$

and

$$
\begin{aligned}
& \left(\int_{2^{k}|z-y| \leq|x-y|<2^{k+1}|z-y|}\left(\left\|F_{t}(x, y)-F_{t}(x, z)\right\|+\left\|F_{t}(y, x)-F_{t}(z, x)\right\|\right)^{q} d y\right)^{1 / q} \\
& \quad \leq C_{k}\left(2^{k}|z-y|\right)^{-n / q^{\prime}}
\end{aligned}
$$

where $1<q^{\prime}<2$ and $1 / q+1 / q^{\prime}=1$.

Definition 2 Let $\varphi$ be a positive, increasing function on $R^{+}$and there exists a constant $D>0$ such that

$$
\varphi(2 t) \leq D \varphi(t) \quad \text { for } t \geq 0
$$

Let $f$ be a locally integrable function on $R^{n}$. Set, for $0 \leq \eta<n$ and $1 \leq p<n / \eta$,

$$
\|f\|_{L^{p, \eta, \varphi}}=\sup _{x \in R^{n}, d>0}\left(\frac{1}{\varphi(d)^{1-p \eta / n}} \int_{Q(x, d)}|f(y)|^{p} d y\right)^{1 / p},
$$

where $Q(x, d)=\left\{y \in R^{n}:|x-y|<d\right\}$. The generalized fractional Morrey space is defined by

$$
L^{p, \eta, \varphi}\left(R^{n}\right)=\left\{f \in L_{\mathrm{loc}}^{1}\left(R^{n}\right):\|f\|_{L^{p, \eta, \varphi}}<\infty\right\} .
$$

We write $L^{p, \eta, \varphi}\left(R^{n}\right)=L^{p, \varphi}\left(R^{n}\right)$ if $\eta=0$, which is the generalized Morrey space. If $\varphi(d)=d^{\delta}$, $\delta>0$, then $L^{p, \varphi}\left(R^{n}\right)=L^{p, \delta}\left(R^{n}\right)$, which is the classical Morrey spaces (see [14, 15]). If $\varphi(d)=1$, then $L^{p, \varphi}\left(R^{n}, w\right)=L^{p}\left(R^{n}\right)$, which is the Lebesgue spaces.

As the Morrey space may be considered as an extension of the Lebesgue space, it is natural and important to study the boundedness of the operator on the Morrey spaces (see $[7,16-19])$. 
It is well known that commutators are of great interest in harmonic analysis and have been widely studied by many authors (see [5, 6]). In [6], Pérez and Trujillo-Gonzalez prove a sharp estimate for the multilinear commutator. The main purpose of this paper is to prove the sharp maximal inequalities for the commutator. As the application, we obtain the $L^{p}$-norm inequality, Morrey and Triebel-Lizorkin spaces boundedness for the commutator.

\section{Theorems}

We shall prove the following theorems.

Theorem 1 Let $T$ be the integral operator as Definition 1 , the sequence $\left\{C_{k}\right\} \in l^{1}, 0<\beta<1$, $q^{\prime} \leq s<\infty$ and $b \in \operatorname{Lip}_{\beta}\left(R^{n}\right)$. Then there exists a constant $C>0$ such that, for any $f \in$ $C_{0}^{\infty}\left(R^{n}\right)$ and $\tilde{x} \in R^{n}$,

$$
M^{\#}\left(T^{b}(f)\right)(\tilde{x}) \leq C\|b\|_{\operatorname{Lip}_{\beta}}\left(M_{\beta, s}(f)(\tilde{x})+M_{\beta, s}(T(f))(\tilde{x})\right) .
$$

Theorem 2 Let $T$ be the integral operator as Definition 1 , the sequence $\left\{2^{k \beta} C_{k}\right\} \in l^{1}, 0<$ $\beta<1, q^{\prime} \leq s<\infty$ and $b \in \operatorname{Lip}_{\beta}\left(R^{n}\right)$. Then there exists a constant $C>0$ such that, for any $f \in C_{0}^{\infty}\left(R^{n}\right)$ and $\tilde{x} \in R^{n}$,

$$
\sup _{Q} \inf _{Q \ni \tilde{x} \in R^{n}} \frac{1}{|Q|^{1+\beta / n}} \int_{Q}\left|T^{b}(f)(x)-c\right| d x \leq C\|b\|_{\operatorname{Lip}_{\beta}}\left(M_{s}(f)(\tilde{x})+M_{s}(T(f))(\tilde{x})\right) .
$$

Theorem 3 Let $T$ be the integral operator as Definition 1 , the sequence $\left\{k C_{k}\right\} \in l^{1}, q^{\prime} \leq s<$ $\infty$ and $b \in B M O\left(R^{n}\right)$. Then there exists a constant $C>0$ such that, for any $f \in C_{0}^{\infty}\left(R^{n}\right)$ and $\tilde{x} \in R^{n}$,

$$
M^{\#}\left(T^{b}(f)\right)(\tilde{x}) \leq C\|b\|_{B M O}\left(M_{s}(f)(\tilde{x})+M_{s}(T(f))(\tilde{x})\right) .
$$

Theorem 4 Let $T$ be the integral operator as Definition 1 , the sequence $\left\{C_{k}\right\} \in l^{1}, 0<\beta<$ $\min \left(1, n / q^{\prime}\right), q^{\prime}<p<n / \beta, 1 / r=1 / p-\beta / n$ and $b \in \operatorname{Lip}_{\beta}\left(R^{n}\right)$. Then $T^{b}$ is bounded from $L^{p}\left(R^{n}\right)$ to $L^{r}\left(R^{n}\right)$.

Theorem 5 Let $T$ be the integral operator as Definition 1 , the sequence $\left\{C_{k}\right\} \in l^{1}, 0<D<$ $2^{n}, 0<\beta<\min \left(1, n / q^{\prime}\right), q^{\prime}<p<n / \beta, 1 / r=1 / p-\beta / n$ and $b \in \operatorname{Lip}_{\beta}\left(R^{n}\right)$. Then $T^{b}$ is bounded from $L^{p, \beta, \varphi}\left(R^{n}\right)$ to $L^{r, \varphi}\left(R^{n}\right)$.

Theorem 6 Let $T$ be the integral operator as Definition 1 , the sequence $\left\{2^{k \beta} C_{k}\right\} \in l^{1}, 0<$ $\beta<\min \left(1, n / q^{\prime}\right), q^{\prime}<p<n / \beta, 1 / r=1 / p-\beta / n$ and $b \in \operatorname{Lip}_{\beta}\left(R^{n}\right)$. Then $T^{b}$ is bounded from $L^{p}\left(R^{n}\right)$ to $\dot{F}_{r}^{\beta, \infty}\left(R^{n}\right)$.

Theorem 7 Let $T$ be the integral operator as Definition 1 , the sequence $\left\{k C_{k}\right\} \in l^{1}$ and $b \in B M O\left(R^{n}\right)$. Then $T^{b}$ is bounded on $L^{p}\left(R^{n}\right)$ for $q^{\prime} \leq p<\infty$.

\section{Proofs of theorems}

To prove the theorems, we need the following lemma. 
Lemma 1 (see [12]) Let $T$ be the integral operator as Definition 1 , the sequence $\left\{C_{k}\right\} \in l^{1}$. Then $T$ is bounded on $L^{p}\left(R^{n}\right)$ for $1<p<\infty$.

Lemma 2 (see [11]) For $0<\beta<1$ and $1<p<\infty$, we have

$$
\begin{aligned}
\|f\|_{\dot{F}_{p}^{\beta, \infty}} & \approx\left\|\sup _{Q \ni \cdot} \frac{1}{|Q|^{1+\beta / n}} \int_{Q}\left|f(x)-f_{Q}\right| d x\right\|_{L^{p}} \\
& \approx\left\|\operatorname{supinf}_{Q \ni \cdot c} \frac{1}{|Q|^{1+\beta / n}} \int_{Q}|f(x)-c| d x\right\|_{L^{p}} .
\end{aligned}
$$

Lemma 3 (see [1]) Let $0<p<\infty$ and $w \in \bigcup_{1 \leq r<\infty} A_{r}$. Then, for any smooth function $f$ for which the left-hand side is finite,

$$
\int_{R^{n}} M(f)(x)^{p} w(x) d x \leq C \int_{R^{n}} M^{\#}(f)(x)^{p} w(x) d x .
$$

Lemma 4 (see [8]) Suppose that $0<\eta<n, 1<s<p<n / \eta$ and $1 / q=1 / p-\eta / n$. Then

$$
\left\|M_{\eta, s}(f)\right\|_{L^{q}} \leq C\|f\|_{L^{p}}
$$

Lemma 5 Let $1<p<\infty, 0<D<2^{n}$. Then, for any smooth function $f$ for which the left-hand side is finite,

$$
\|M(f)\|_{L^{p, \varphi}} \leq C\left\|M^{\#}(f)\right\|_{L^{p, \varphi}} .
$$

Proof For any cube $Q=Q\left(x_{0}, d\right)$ in $R^{n}$, we know $M\left(\chi_{Q}\right) \in A_{1}$ for any cube $Q=Q(x, d)$ by [20]. Noticing that $M\left(\chi_{Q}\right) \leq 1$ and $M\left(\chi_{Q}\right)(x) \leq d^{n} /\left(\left|x-x_{0}\right|-d\right)^{n}$ if $x \in Q^{c}$, by Lemma 3 , we have, for $f \in L^{p, \varphi}\left(R^{n}\right)$,

$$
\begin{aligned}
\int_{Q} M(f)(x)^{p} d x= & \int_{R^{n}} M(f)(x)^{p} \chi_{Q}(x) d x \\
\leq & \int_{R^{n}} M(f)(x)^{p} M\left(\chi_{Q}\right)(x) d x \\
\leq & C \int_{R^{n}} M^{\#}(f)(x)^{p} M\left(\chi_{Q}\right)(x) d x \\
= & C\left(\int_{Q} M^{\#}(f)(x)^{p} M\left(\chi_{Q}\right)(x) d x\right. \\
& \left.+\sum_{k=0}^{\infty} \int_{2^{k+1} Q \backslash 2^{k} Q} M^{\#}(f)(x)^{p} M\left(\chi_{Q}\right)(x) d x\right) \\
\leq & C\left(\int_{Q} M^{\#}(f)(x)^{p} d x+\sum_{k=0}^{\infty} \int_{2^{k+1} Q \backslash 2^{k} Q} M^{\#}(f)(x)^{p} \frac{|Q|}{\left|2^{k+1} Q\right|} d x\right) \\
\leq & C\left(\int_{Q} M^{\#}(f)(x)^{p} d x+\sum_{k=0}^{\infty} \int_{2^{k+1} Q} M^{\#}(f)(x)^{p} 2^{-k n} d y\right) \\
\leq & C\left\|M^{\#}(f)\right\|_{L^{p, \varphi}}^{p} \sum_{k=0}^{\infty} 2^{-k n} \varphi\left(2^{k+1} d\right)
\end{aligned}
$$




$$
\begin{aligned}
& \leq C\left\|M^{\#}(f)\right\|_{L^{p, \varphi}}^{p} \sum_{k=0}^{\infty}\left(2^{-n} D\right)^{k} \varphi(d) \\
& \leq C\left\|M^{\#}(f)\right\|_{L^{p, \varphi}}^{p} \varphi(d),
\end{aligned}
$$

thus

$$
\left(\frac{1}{\varphi(d)} \int_{Q} M(f)(x)^{p} d x\right)^{1 / p} \leq C\left(\frac{1}{\varphi(d)} \int_{Q} M^{\#}(f)(x)^{p} d x\right)^{1 / p}
$$

and

$$
\|M(f)\|_{L^{p, \varphi}} \leq C\left\|M^{\#}(f)\right\|_{L^{p, \varphi}} \cdot
$$

This finishes the proof.

Lemma 6 Let $T$ be the integral operator as Definition 1, $0 \leq \eta<n, 0<D<2^{n}$ and $1 \leq p<\infty$. Then

$$
\|T(f)\|_{L^{p, \eta, \varphi}} \leq C\|f\|_{L^{p, \eta, \varphi}} .
$$

Lemma 7 Let $0<D<2^{n}, 0<\eta<n, 1 \leq s<p<n / \eta$ and $1 / q=1 / p-\eta / n$. Then

$$
\left\|M_{\eta, s}(f)\right\|_{L^{q, \varphi}} \leq C\|f\|_{L^{p, \eta, \varphi}} .
$$

The proofs of the two lemmas are similar to that of Lemma 5 by Lemmas 1 and 4, we omit the details.

Proof of Theorem 1 It suffices to prove for $f \in C_{0}^{\infty}\left(R^{n}\right)$ and some constant $C_{0}$, the following inequality holds:

$$
\frac{1}{|Q|} \int_{Q}\left|T^{b}(f)(x)-C_{0}\right| d x \leq C\|b\|_{\operatorname{Lip}_{\beta}}\left(M_{\beta, s}(f)(\tilde{x})+M_{\beta, s}(T(f))(\tilde{x})\right) .
$$

Fix a cube $Q=Q\left(x_{0}, d\right)$ and $\tilde{x} \in Q$. Write, for $f_{1}=f \chi_{2 Q}$ and $f_{2}=f \chi_{(2 Q)^{c}}$,

$$
F_{t}^{b}(f)(x)=\left(b(x)-b_{2 Q}\right) F_{t}(f)(x)-F_{t}\left(\left(b-b_{2 Q}\right) f_{1}\right)(x)-F_{t}\left(\left(b-b_{2 Q}\right) f_{2}\right)(x) .
$$

Then

$$
\begin{aligned}
\frac{1}{|Q|} \int_{Q}\left\|F_{t}^{b}(f)(x)-F_{t}\left(\left(b_{2 Q}-b\right) f_{2}\right)\left(x_{0}\right)\right\| d x \\
\leq \frac{1}{|Q|} \int_{Q}\left\|\left(b(x)-b_{2 Q}\right) F_{t}(f)(x)\right\| d x+\frac{1}{|Q|} \int_{Q}\left\|F_{t}\left(\left(b-b_{2 Q}\right) f_{1}\right)(x)\right\| d x \\
\quad+\frac{1}{|Q|} \int_{Q}\left\|F_{t}\left(\left(b-b_{2 Q}\right) f_{2}\right)(x)-F_{t}\left(\left(b-b_{2 Q}\right) f_{2}\right)\left(x_{0}\right)\right\| d x \\
=I_{1}+I_{2}+I_{3} .
\end{aligned}
$$


For $I_{1}$, by Hölder's inequality and Lemma 2, we obtain

$$
\begin{aligned}
I_{1} & \leq \frac{C}{|Q|} \sup _{x \in 2 Q}\left|b(x)-b_{2 Q}\right||Q|^{1-1 / s}\left(\int_{Q}|T(f)(x)|^{s} d x\right)^{1 / s} \\
& \leq C|Q|^{-1 / s}\|b\|_{\operatorname{Lip}_{\beta}}|2 Q|^{\beta / n}|2 Q|^{1 / s-\beta / n}\left(\frac{1}{|Q|^{1-s \beta / n}} \int_{Q}|T(f)(x)|^{s} d x\right)^{1 / s} \\
& \leq C\|b\|_{\operatorname{Lip}_{\beta}} M_{\beta, s}(T(f))(\tilde{x}) .
\end{aligned}
$$

For $I_{2}$, by the boundedness of $T$, we get

$$
\begin{aligned}
I_{2} & \leq\left(\frac{1}{|Q|} \int_{R^{n}}\left|T\left(\left(b-b_{2 Q}\right) f_{1}\right)(x)\right|^{s} d x\right)^{1 / s} \\
& \leq C\left(\frac{1}{|Q|} \int_{R^{n}}\left|\left(b(x)-b_{2 Q}\right) f_{1}(x)\right|^{s} d x\right)^{1 / s} \\
& \leq\left. C|Q|^{-1 / s}|2 Q|^{\beta / n}|| 2 Q\right|^{1 / s-\beta / n}\left(\frac{1}{|2 Q|^{1-s \beta / n}} \int_{2 Q}|f(x)|^{s} d x\right)^{1 / s} \\
& \leq C\|b\|_{\operatorname{Lip}_{\beta}} M_{\beta, s}(f)(\tilde{x}) .
\end{aligned}
$$

For $I_{3}$, recalling that $s>q^{\prime}$, we have

$$
\begin{aligned}
& I_{3} \leq \frac{1}{|Q|} \int_{Q} \int_{(2 Q)^{c}}\left|b(y)-b_{2 Q}\right||f(y)|\left\|F_{t}(x, y)-F_{t}\left(x_{0}, y\right)\right\| d y d x \\
& \leq \frac{1}{|Q|} \int_{Q} \sum_{k=1}^{\infty} \int_{2^{k} d \leq\left|y-x_{0}\right|<2^{k+1} d}\left\|F_{t}(x, y)-F_{t}\left(x_{0}, y\right)\right\|\left|b(y)-b_{2^{k+1} Q}\right||f(y)| d y d x \\
& +\frac{1}{|Q|} \int_{Q} \sum_{k=1}^{\infty} \int_{2^{k} d \leq\left|y-x_{0}\right|<2^{k+1} d}\left\|F_{t}(x, y)-F_{t}\left(x_{0}, y\right)\right\|\left|b_{2^{k+1} Q}-b_{2 Q}\right||f(y)| d y d x \\
& \leq \frac{C}{|Q|} \int_{Q} \sum_{k=1}^{\infty}\left(\int_{2^{k} d \leq\left|y-x_{0}\right|<2^{k+1} d}\left\|F_{t}(x, y)-F_{t}\left(x_{0}, y\right)\right\|^{q} d y\right)^{1 / q} \\
& \times \sup _{y \in 2^{k+1} Q}\left|b(y)-b_{2^{k+1} Q}\right|\left(\int_{2^{k+1} Q}|f(y)|^{q^{\prime}} d y\right)^{1 / q^{\prime}} d x \\
& +\frac{C}{|Q|} \int_{Q} \sum_{k=1}^{\infty}\left|b_{2^{k+1} Q}-b_{2 Q}\right|\left(\int_{2^{k} d \leq\left|y-x_{0}\right|<2^{k+1} d}\left\|F_{t}(x, y)-F_{t}\left(x_{0}, y\right)\right\|^{q} d y\right)^{1 / q} \\
& \times\left(\int_{2^{k+1} Q}|f(y)|^{q^{\prime}} d y\right)^{1 / q^{\prime}} d x \\
& \leq C \sum_{k=1}^{\infty} C_{k}\left(2^{k} d\right)^{-n / q^{\prime}}\left|2^{k+1} Q\right|^{\beta / n}\|b\|_{\operatorname{Lip}_{\beta}}\left|2^{k+1} Q\right|^{1 / q^{\prime}-1 / s}\left|2^{k+1} Q\right|^{1 / s-\beta / n} \\
& \times\left(\frac{1}{\left|2^{k+1} Q\right|^{1-s \beta / n}} \int_{2^{k+1} Q}|f(y)|^{s} d y\right)^{1 / s} \\
& +C \sum_{k=1}^{\infty}\|b\|_{\operatorname{Lip}_{\beta}}\left|2^{k} Q\right|^{\beta / n} C_{k}\left(2^{k} d\right)^{-n / q^{\prime}}\left|2^{k+1} Q\right|^{1 / q^{\prime}-1 / s}\left|2^{k+1} Q\right|^{1 / s-\beta / n}
\end{aligned}
$$




$$
\begin{aligned}
& \times\left(\frac{1}{\left|2^{k+1} Q\right|^{1-s \beta / n}} \int_{2^{k+1} Q}|f(y)|^{s} d y\right)^{1 / s} \\
\leq & C\|b\|_{\operatorname{Lip}_{\beta}} M_{\beta, s}(f)(\tilde{x}) \sum_{k=1}^{\infty} C_{k} \\
\leq & C\|b\|_{\operatorname{Lip}_{\beta}} M_{\beta, s}(f)(\tilde{x}) .
\end{aligned}
$$

This completes the proof of Theorem 1.

Proof of Theorem 2 It suffices to prove for $f \in C_{0}^{\infty}\left(R^{n}\right)$ and some constant $C_{0}$, the following inequality holds:

$$
\frac{1}{|Q|^{1+\beta / n}} \int_{Q}\left|T^{b}(f)(x)-C_{0}\right| d x \leq C\|b\|_{\operatorname{Lip}_{\beta}}\left(M_{s}(f)(\tilde{x})+M_{s}(T(f))(\tilde{x})\right) .
$$

Fix a cube $Q=Q\left(x_{0}, d\right)$ and $\tilde{x} \in Q$. Write, for $f_{1}=f \chi_{2 Q}$ and $f_{2}=f \chi_{(2 Q) c}$,

$$
F_{t}^{b}(f)(x)=\left(b(x)-b_{2 Q}\right) F_{t}(f)(x)-F_{t}\left(\left(b-b_{2 Q}\right) f_{1}\right)(x)-F_{t}\left(\left(b-b_{2 Q}\right) f_{2}\right)(x) .
$$

Then

$$
\begin{aligned}
& \frac{1}{|Q|^{1+\beta / n}} \int_{Q}\left\|F_{t}^{b}(f)(x)-F_{t}\left(\left(b_{2 Q}-b\right) f_{2}\right)\left(x_{0}\right)\right\| d x \\
& \leq \frac{1}{|Q|^{1+\beta / n}} \int_{Q}\left\|\left(b(x)-b_{2 Q}\right) F_{t}(f)(x)\right\| d x+\frac{1}{|Q|^{1+\beta / n}} \int_{Q}\left\|F_{t}\left(\left(b-b_{2 Q}\right) f_{1}\right)(x)\right\| d x \\
& \quad+\frac{1}{|Q|^{1+\beta / n}} \int_{Q}\left\|F_{t}\left(\left(b-b_{2 Q}\right) f_{2}\right)(x)-F_{t}\left(\left(b-b_{2 Q}\right) f_{2}\right)\left(x_{0}\right)\right\| d x \\
& =I I_{1}+I I_{2}+I I_{3} .
\end{aligned}
$$

By using the same argument as in the proof of Theorem 1, we get

$$
\begin{aligned}
I I_{1} & \leq \frac{C}{|Q|^{1+\beta / n}} \sup _{x \in 2 Q}\left|b(x)-b_{2 Q}\right||Q|^{1-1 / s}\left(\int_{Q}|T(f)(x)|^{s} d x\right)^{1 / s} \\
& \leq C\|b\|_{\operatorname{Lip}_{\beta}}|2 Q|^{\beta / n}|Q|^{-1 / s}|Q|^{1 / s-\beta / n}\left(\frac{1}{|Q|} \int_{Q}|T(f)(x)|^{s} d x\right)^{1 / s} \\
& \leq C\|b\|_{\operatorname{Lip}_{\beta}} M_{s}(T(f))(\tilde{x}), \\
I I_{2} & \leq \frac{1}{|Q|^{1+\beta / n}}|Q|^{1-1 / s}\left(\int_{R^{n}}\left|T\left(\left(b-b_{2 Q}\right) f_{1}\right)(x)\right|^{s} d x\right)^{1 / s} \\
& \leq \frac{C}{|Q|^{1+\beta / n}}|Q|^{1-1 / s}\left(\int_{R^{n}}\left|\left(b(x)-b_{2 Q}\right) f_{1}(x)\right|^{s} d x\right)^{1 / s} \\
& \leq \frac{C}{|Q|^{1+\beta / n}}|Q|^{1-1 / s}|2 Q|^{\beta / n}|2 Q|^{1 / s}\left(\frac{1}{|2 Q|} \int_{2 Q}|f(x)|^{s} d x\right)^{1 / s} \\
& \leq C\|b\|_{\operatorname{Lip}_{\beta}} M_{s}(f)(\tilde{x}), \\
I I_{3} & \leq \frac{1}{|Q|^{1+\beta / n}} \int_{Q} \int_{(2 Q)}\left|b(y)-b_{2 Q}\right||f(y)|\left\|F_{t}(x, y)-F_{t}\left(x_{0}, y\right)\right\| d y d x
\end{aligned}
$$




$$
\begin{aligned}
& \leq \frac{1}{|Q|^{1+\beta / n}} \int_{Q} \sum_{k=1}^{\infty} \int_{2^{k} d \leq\left|y-x_{0}\right|<2^{k+1} d}\left\|F_{t}(x, y)-F_{t}\left(x_{0}, y\right)\right\|\left|b(y)-b_{2^{k+1} Q}\right||f(y)| d y d x \\
& +\frac{1}{|Q|^{1+\beta / n}} \int_{Q} \sum_{k=1}^{\infty} \int_{2^{k} d \leq\left|y-x_{0}\right|<2^{k+1} d}\left\|F_{t}(x, y)-F_{t}\left(x_{0}, y\right)\right\|\left|b_{2^{k+1} Q}-b_{2 Q}\right||f(y)| d y d x \\
& \leq \frac{C}{|Q|^{1+\beta / n}} \int_{Q} \sum_{k=1}^{\infty}\left(\int_{2^{k} d \leq\left|y-x_{0}\right|<2^{k+1} d}\left\|F_{t}(x, y)-F_{t}\left(x_{0}, y\right)\right\|^{q} d y\right)^{1 / q} \\
& \times \sup _{y \in 2^{k+1} Q}\left|b(y)-b_{2^{k+1} Q}\right|\left(\int_{2^{k+1} Q}|f(y)|^{q^{\prime}} d y\right)^{1 / q^{\prime}} d x \\
& +\frac{C}{|Q|^{1+\beta / n}} \int_{Q} \sum_{k=1}^{\infty}\left|b_{2^{k+1} Q}-b_{2 Q}\right|\left(\int_{2^{k} d \leq\left|y-x_{0}\right|<2^{k+1} d}\left\|F_{t}(x, y)-F_{t}\left(x_{0}, y\right)\right\|^{q} d y\right)^{1 / q} \\
& \times\left(\int_{2^{k+1} Q}|f(y)|^{q^{\prime}} d y\right)^{1 / q^{\prime}} d x \\
& \leq C|Q|^{-\beta / n} \sum_{k=1}^{\infty} C_{k}\left(2^{k} d\right)^{-n / q^{\prime}}\left|2^{k+1} Q\right|^{\beta / n}\|b\|_{\operatorname{Lip}_{\beta}}\left|2^{k+1} Q\right|^{1 / q^{\prime}} \\
& \times\left(\frac{1}{\left|2^{k+1} Q\right|} \int_{2^{k+1} Q}|f(y)|^{s} d y\right)^{1 / s} \\
& +C|Q|^{-\beta / n} \sum_{k=1}^{\infty}\|b\|_{\operatorname{Lip}_{\beta}}\left|2^{k} Q\right|^{\beta / n} C_{k}\left(2^{k} d\right)^{-n / q^{\prime}}\left|2^{k+1} Q\right|^{1 / q^{\prime}} \\
& \times\left(\frac{1}{\left|2^{k+1} Q\right|} \int_{2^{k+1} Q}|f(x)|^{s} d x\right)^{1 / s} \\
& \leq C\|b\|_{\operatorname{Lip}_{\beta}} M_{s}(f)(\tilde{x}) \sum_{k=1}^{\infty} 2^{k \beta} C_{k} \\
& \leq C\|b\|_{\operatorname{Lip}_{\beta}} M_{s}(f)(\tilde{x}) .
\end{aligned}
$$

This completes the proof of Theorem 2 .

Proof of Theorem 3 It suffices to prove for $f \in C_{0}^{\infty}\left(R^{n}\right)$ and some constant $C_{0}$, the following inequality holds:

$$
\frac{1}{|Q|} \int_{Q}\left|T^{b}(f)(x)-C_{0}\right| d x \leq C\|b\|_{B M O}\left(M_{s}(f)(\tilde{x})+M_{s}(T(f))(\tilde{x})\right) .
$$

Fix a cube $Q=Q\left(x_{0}, d\right)$ and $\tilde{x} \in Q$. Write, for $f_{1}=f \chi_{2 Q}$ and $f_{2}=f \chi_{(2 Q)}$,

$$
F_{t}^{b}(f)(x)=\left(b(x)-b_{2 Q}\right) F_{t}(f)(x)-F_{t}\left(\left(b-b_{2 Q}\right) f_{1}\right)(x)-F_{t}\left(\left(b-b_{2 Q}\right) f_{2}\right)(x) .
$$

Then

$$
\begin{aligned}
& \frac{1}{|Q|} \int_{Q}\left\|F_{t}^{b}(f)(x)-F_{t}\left(\left(b_{2 Q}-b\right) f_{2}\right)\left(x_{0}\right)\right\| d x \\
& \quad \leq \frac{1}{|Q|} \int_{Q}\left\|\left(b(x)-b_{2 Q}\right) F_{t}(f)(x)\right\| d x+\frac{1}{|Q|} \int_{Q}\left\|F_{t}\left(\left(b-b_{2 Q}\right) f_{1}\right)(x)\right\| d x
\end{aligned}
$$




$$
\begin{aligned}
& +\frac{1}{|Q|} \int_{Q}\left\|F_{t}\left(\left(b-b_{2 Q}\right) f_{2}\right)(x)-F_{t}\left(\left(b-b_{2 Q}\right) f_{2}\right)\left(x_{0}\right)\right\| d x \\
= & I I I_{1}+I I I_{2}+I I I_{3} .
\end{aligned}
$$

For $I I I_{1}$, by Hölder's inequality, we get

$$
\begin{aligned}
I I I_{1} & \leq\left(\frac{1}{|Q|} \int_{Q}\left|b(x)-b_{2 Q}\right|^{s^{\prime}} d x\right)^{1 / s^{\prime}}\left(\frac{1}{|Q|} \int_{Q}|T(f)(x)|^{s} d x\right)^{1 / s} \\
& \leq C\|b\|_{B M O} M_{s}(T(f))(\tilde{x}) .
\end{aligned}
$$

For $I I I_{2}$, choose $1<r<s$, by Hölder's inequality and the boundedness of $T$, we obtain

$$
\begin{aligned}
I I I_{2} & \leq\left(\frac{1}{|Q|} \int_{R^{n}}\left|T\left(\left(b-b_{2 Q}\right) f_{1}\right)(x)\right|^{r} d x\right)^{1 / r} \\
& \leq C|Q|^{-1 / r}\left(\int_{R^{n}}\left|\left(b-b_{2 Q}\right) f_{1}(x)\right|^{r} d x\right)^{1 / r} \\
& \leq C|Q|^{-1 / r}\left(\int_{2 Q}\left|b(x)-b_{2 Q}\right|^{s r /(s-r)} d x\right)^{(s-r) / s r}\left(\int_{2 Q}|f(x)|^{s} d x\right)^{1 / s} \\
& \leq C\|b\|_{B M O}\left(\frac{1}{|2 Q|} \int_{2 Q}|f(x)|^{s} d x\right)^{1 / s} \\
& \leq C\|b\|_{B M O} M_{s}(f)(\tilde{x}) .
\end{aligned}
$$

For $I I I_{3}$, recalling that $s>q^{\prime}$, taking $1<p<\infty, 1<r<s$ with $1 / p+1 / q+1 / r=1$, by Hölder's inequality, we obtain

$$
\begin{aligned}
I I_{3} \leq & \frac{1}{|Q|} \int_{Q} \int_{(2 Q) c^{c}}\left|b(y)-b_{2 Q}\right||f(y)|\left\|F_{t}(x, y)-F_{t}\left(x_{0}, y\right)\right\| d y d x \\
\leq & \frac{1}{|Q|} \int_{Q} \sum_{k=1}^{\infty} \int_{2^{k} d \leq\left|y-x_{0}\right|<2^{k+1} d}\left\|F_{t}(x, y)-F_{t}\left(x_{0}, y\right)\right\|\left|b(y)-b_{2 Q}\right||f(y)| d y d x \\
\leq & \frac{1}{|Q|} \int_{Q} \sum_{k=1}^{\infty}\left(\int_{2^{k} d \leq\left|y-x_{0}\right|<2^{k+1} d}\left\|F_{t}(x, y)-F_{t}\left(x_{0}, y\right)\right\|^{q} d y\right)^{1 / q} \\
& \times\left(\int_{2^{k+1} Q}\left|b(x)-b_{2 Q}\right|^{p} d x\right)^{1 / p}\left(\int_{2^{k+1} Q}|f(y)|^{r} d y\right)^{1 / r} d x \\
\leq & C \sum_{k=1}^{\infty} k\left(2^{k} d\right)^{n / q^{\prime}}\left(\int_{2^{k} d \leq\left|y-x_{0}\right|<2^{k+1} d}\left\|K(x, y)-K\left(x_{0}, y\right)\right\|^{q} d y\right)^{1 / q} \\
& \times\|b\|_{B M O}\left(\frac{1}{\left|2^{k+1} Q\right|} \int_{2^{k+1} Q}|f(y)|^{s} d y\right)^{1 / s} \\
\leq & C\|b\|_{B M O} M_{s}(f)(\tilde{x}) \sum_{k=1}^{\infty} k C_{k} \\
\leq & C\|b\|_{B M O} M_{s}(f)(\tilde{x}) .
\end{aligned}
$$

This completes the proof of Theorem 3. 
Proof of Theorem 4 Choose $q^{\prime}<s<p$ in Theorem 1, we have, by Lemmas 1, 4, and 5,

$$
\begin{aligned}
\left\|T^{b}(f)\right\|_{L^{r}} & \leq\left\|M\left(T^{b}(f)\right)\right\|_{L^{r}} \leq C\left\|M^{\#}\left(T^{b}(f)\right)\right\|_{L^{r}} \\
& \leq C\|b\|_{\operatorname{Lip}_{\beta}}\left(\left\|M_{\beta, s}(T(f))\right\|_{L^{r}}+\left\|M_{\beta, s}(f)\right\|_{L^{r}}\right) \\
& \leq C\|b\|_{\operatorname{Lip}_{\beta}}\left(\|T(f)\|_{L^{p}}+\|f\|_{L^{p}}\right) \\
& \leq C\|b\|_{\operatorname{Lip}_{\beta}}\|f\|_{L^{p}} .
\end{aligned}
$$

This completes the proof of Theorem 4 .

Proof of Theorem 5 Choose $q^{\prime}<s<p$ in Theorem 1, we have, by Lemmas 5-7,

$$
\begin{aligned}
\left\|T^{b}(f)\right\|_{L^{r, \varphi}} & \leq\left\|M\left(T^{b}(f)\right)\right\|_{L^{r, \varphi}} \leq C\left\|M^{\#}\left(T^{b}(f)\right)\right\|_{L^{r, \varphi}} \\
& \leq C\|b\|_{\operatorname{Lip}_{\beta}}\left(\left\|M_{\beta, s}(T(f))\right\|_{L^{r, \varphi}}+\left\|M_{\beta, s}(f)\right\|_{L^{r, \varphi}}\right) \\
& \leq C\|b\|_{\operatorname{Lip}_{\beta}}\left(\|T(f)\|_{L^{p, \beta, \varphi}}+\|f\|_{L^{p, \beta, \varphi}}\right) \\
& \leq C\|b\|_{\operatorname{Lip}_{\beta}}\|f\|_{L^{p, \varphi}} .
\end{aligned}
$$

This completes the proof of Theorem 5 .

Proof Theorem 6 Choose $q^{\prime}<s<p$ in Theorem 2. By using Lemma 3, we obtain

$$
\begin{aligned}
\left\|T^{b}(f)\right\|_{\dot{F}_{r}^{\beta, \infty}} & \leq C\left\|\sup _{Q \ni \cdot} \frac{1}{|Q|^{1+\beta / n}} \int_{Q}\left|T^{b}(f)(x)-T\left(\left(b_{2 Q}-b\right) f_{2}\right)\left(x_{0}\right)\right| d x\right\|_{L^{r}} \\
& \leq C\|b\|_{\operatorname{Lip}_{\beta}}\left(\left\|M_{s}(T(f))\right\|_{L^{r}}+\left\|M_{s}(f)\right\|_{L^{r}}\right) \\
& \leq C\|b\|_{\operatorname{Lip}_{\beta}}\left(\|T(f)\|_{L^{p}}+\|f\|_{L^{p}}\right) \\
& \leq C\|b\|_{\operatorname{Lip}_{\beta}}\|f\|_{L^{p}} .
\end{aligned}
$$

This completes the proof of the theorem.

Proof of Theorem 7 Choose $q^{\prime} \leq s<p$ in Theorem 3, we have

$$
\begin{aligned}
\left\|T^{b}(f)\right\|_{L^{p}} & \leq\left\|M\left(T^{b}(f)\right)\right\|_{L^{p}} \leq C\left\|M^{\#}\left(T^{b}(f)\right)\right\|_{L^{p}} \\
& \leq C\|b\|_{B M O}\left(\left\|M_{s}(T(f))\right\|_{L^{p}}+\left\|M_{s}(f)\right\|_{L^{p}}\right) \\
& \leq C\|b\|_{B M O}\left(\|T(f)\|_{L^{p}}+\|f\|_{L^{p}}\right) \\
& \leq C\|b\|_{B M O}\|f\|_{L^{p}} .
\end{aligned}
$$

This completes the proof of the theorem.

\section{Applications}

In this section we shall apply Theorems 1-6 of the paper to some particular operators such as the Littlewood-Paley operators, Marcinkiewicz operator and Bochner-Riesz operator.

Application 1 Littlewood-Paley operators.

Fixed $\varepsilon>0$ and $\mu>(3 n+2) / n$. Let $\psi$ be a fixed function which satisfies: 
(1) $\int_{R^{n}} \psi(x) d x=0$,

(2) $|\psi(x)| \leq C(1+|x|)^{-(n+1)}$,

(3) $|\psi(x+y)-\psi(x)| \leq C|y|^{\varepsilon}(1+|x|)^{-(n+1+\varepsilon)}$ when $2|y|<|x|$.

We denote $\Gamma(x)=\left\{(y, t) \in R_{+}^{n+1}:|x-y|<t\right\}$ and the characteristic function of $\Gamma(x)$ by $\chi_{\Gamma(x)}$. The Littlewood-Paley commutators are defined by

$$
\begin{aligned}
& g_{\psi}^{b}(f)(x)=\left(\int_{0}^{\infty}\left|F_{t}^{b}(f)(x)\right|^{2} \frac{d t}{t}\right)^{1 / 2}, \\
& S_{\psi}^{b}(f)(x)=\left[\iint_{\Gamma(x)}\left|F_{t}^{b}(f)(x, y)\right|^{2} \frac{d y d t}{t^{n+1}}\right]^{1 / 2},
\end{aligned}
$$

and

$$
g_{\mu}^{b}(f)(x)=\left[\iint_{R_{+}^{n+1}}\left(\frac{t}{t+|x-y|}\right)^{n \mu}\left|F_{t}^{b}(f)(x, y)\right|^{2} \frac{d y d t}{t^{n+1}}\right]^{1 / 2},
$$

where

$$
\begin{aligned}
& F_{t}^{b}(f)(x)=\int_{R^{n}}(b(x)-b(y)) \psi_{t}(x-y) f(y) d y, \\
& F_{t}^{b}(f)(x, y)=\int_{R^{n}}(b(x)-b(z)) f(z) \psi_{t}(y-z) d z
\end{aligned}
$$

and $\psi_{t}(x)=t^{-n} \psi(x / t)$ for $t>0$. Set $F_{t}(f)(y)=f * \psi_{t}(y)$. We also define

$$
\begin{aligned}
& g_{\psi}(f)(x)=\left(\int_{0}^{\infty}\left|F_{t}(f)(x)\right|^{2} \frac{d t}{t}\right)^{1 / 2}, \\
& S_{\psi}(f)(x)=\left(\iint_{\Gamma(x)}\left|F_{t}(f)(y)\right|^{2} \frac{d y d t}{t^{n+1}}\right)^{1 / 2}
\end{aligned}
$$

and

$$
g_{\mu}(f)(x)=\left(\iint_{R_{+}^{n+1}}\left(\frac{t}{t+|x-y|}\right)^{n \mu}\left|F_{t}(f)(y)\right|^{2} \frac{d y d t}{t^{n+1}}\right)^{1 / 2},
$$

which are the Littlewood-Paley operators (see [3]). Let $H$ be the space

$$
H=\left\{h:\|h\|=\left(\int_{0}^{\infty}|h(t)|^{2} d t / t\right)^{1 / 2}<\infty\right\}
$$

or

$$
H=\left\{h:\|h\|=\left(\iint_{R_{+}^{n+1}}|h(y, t)|^{2} d y d t / t^{n+1}\right)^{1 / 2}<\infty\right\},
$$

then, for each fixed $x \in R^{n}, F_{t}^{b}(f)(x)$ and $F_{t}^{b}(f)(x, y)$ may be viewed as the mapping from $[0,+\infty)$ to $H$, and it is clear that

$$
g_{\psi}^{b}(f)(x)=\left\|F_{t}^{b}(f)(x)\right\|, \quad g_{\psi}(f)(x)=\left\|F_{t}(f)(x)\right\|,
$$




$$
S_{\psi}^{b}(f)(x)=\left\|\chi_{\Gamma(x)} F_{t}^{b}(f)(x, y)\right\|, \quad S_{\psi}(f)(x)=\left\|\chi_{\Gamma(x)} F_{t}(f)(y)\right\|
$$

and

$$
g_{\mu}^{b}(f)(x)=\left\|\left(\frac{t}{t+|x-y|}\right)^{n \mu / 2} F_{t}^{b}(f)(x, y)\right\|, \quad g_{\mu}(f)(x)=\left\|\left(\frac{t}{t+|x-y|}\right)^{n \mu / 2} F_{t}(f)(y)\right\| .
$$

It is easily to see that $g_{\psi}^{b}, S_{\psi}^{b}$, and $g_{\mu}^{b}$ satisfy the conditions of Theorems 1-6 (see [21-23]), thus Theorems 1-6 hold for $g_{\psi}^{b}, S_{\psi}^{b}$, and $g_{\mu}^{b}$.

Application 2 Marcinkiewicz operators.

Fixed $\lambda>\max (1,2 n /(n+2))$ and $0<\gamma \leq 1$. Let $\Omega$ be homogeneous of degree zero on $R^{n}$ with $\int_{S^{n-1}} \Omega\left(x^{\prime}\right) d \sigma\left(x^{\prime}\right)=0$. Assume that $\Omega \in \operatorname{Lip}_{\gamma}\left(S^{n-1}\right)$. The Marcinkiewicz commutators are defined by

$$
\begin{aligned}
& \mu_{\Omega}^{b}(f)(x)=\left(\int_{0}^{\infty}\left|F_{t}^{b}(f)(x)\right|^{2} \frac{d t}{t^{3}}\right)^{1 / 2}, \\
& \mu_{S}^{b}(f)(x)=\left[\iint_{\Gamma(x)}\left|F_{t}^{b}(f)(x, y)\right|^{2} \frac{d y d t}{t^{n+3}}\right]^{1 / 2},
\end{aligned}
$$

and

$$
\mu_{\lambda}^{b}(f)(x)=\left[\iint_{R_{+}^{n+1}}\left(\frac{t}{t+|x-y|}\right)^{n \lambda}\left|F_{t}^{b}(f)(x, y)\right|^{2} \frac{d y d t}{t^{n+3}}\right]^{1 / 2},
$$

where

$$
F_{t}^{b}(f)(x)=\int_{|x-y| \leq t}(b(x)-b(y)) \frac{\Omega(x-y)}{|x-y|^{n-1}} f(y) d y
$$

and

$$
F_{t}^{b}(f)(x, y)=\int_{|y-z| \leq t}(b(x)-b(z)) \frac{\Omega(y-z)}{|y-z|^{n-1}} f(z) d z .
$$

Set

$$
F_{t}(f)(x)=\int_{|x-y| \leq t} \frac{\Omega(x-y)}{|x-y|^{n-1}} f(y) d y .
$$

We also define

$$
\begin{aligned}
& \mu_{\Omega}(f)(x)=\left(\int_{0}^{\infty}\left|F_{t}(f)(x)\right|^{2} \frac{d t}{t^{3}}\right)^{1 / 2}, \\
& \mu_{S}(f)(x)=\left(\iint_{\Gamma(x)}\left|F_{t}(f)(y)\right|^{2} \frac{d y d t}{t^{n+3}}\right)^{1 / 2},
\end{aligned}
$$

and

$$
\mu_{\lambda}(f)(x)=\left(\iint_{R_{+}^{n+1}}\left(\frac{t}{t+|x-y|}\right)^{n \lambda}\left|F_{t}(f)(y)\right|^{2} \frac{d y d t}{t^{n+3}}\right)^{1 / 2},
$$


which are the Marcinkiewicz operators (see [24]). Let $H$ be the space

$$
H=\left\{h:\|h\|=\left(\int_{0}^{\infty}|h(t)|^{2} d t / t^{3}\right)^{1 / 2}<\infty\right\}
$$

or

$$
H=\left\{h:\|h\|=\left(\iint_{R_{+}^{n+1}}|h(y, t)|^{2} d y d t / t^{n+3}\right)^{1 / 2}<\infty\right\} .
$$

Then it is clear that

$$
\begin{aligned}
& \mu_{\Omega}^{b}(f)(x)=\left\|F_{t}^{b}(f)(x)\right\|, \quad \mu_{\Omega}(f)(x)=\left\|F_{t}(f)(x)\right\|, \\
& \mu_{S}^{b}(f)(x)=\left\|\chi_{\Gamma(x)} F_{t}^{b}(f)(x, y)\right\|, \quad \mu_{S}(f)(x)=\left\|\chi_{\Gamma(x)} F_{t}(f)(y)\right\|,
\end{aligned}
$$

and

$$
\mu_{\lambda}^{b}(f)(x)=\left\|\left(\frac{t}{t+|x-y|}\right)^{n \lambda / 2} F_{t}^{b}(f)(x, y)\right\|, \quad \mu_{\lambda}(f)(x)=\left\|\left(\frac{t}{t+|x-y|}\right)^{n \lambda / 2} F_{t}(f)(y)\right\| .
$$

It is easy to see that $\mu_{\Omega}^{b}, \mu_{S}^{b}$, and $\mu_{\lambda}^{b}$ satisfy the conditions of Theorems 1-6 (see [21-24]), thus Theorems 1-6 hold for $\mu_{\Omega}^{b}, \mu_{S}^{b}$, and $\mu_{\lambda}^{b}$.

Application 3 Bochner-Riesz operator.

Let $\delta>(n-1) / 2, B_{t}^{\delta}(\hat{f})(\xi)=\left(1-t^{2}|\xi|^{2}\right)_{+}^{\delta} \hat{f}(\xi)$ and $B_{t}^{\delta}(z)=t^{-n} B^{\delta}(z / t)$ for $t>0$. Set

$$
F_{\delta, t}^{b}(f)(x)=\int_{R^{n}}(b(x)-b(y)) B_{t}^{\delta}(x-y) f(y) d y .
$$

The maximal Bochner-Riesz commutator is defined by

$$
B_{\delta, *}^{b}(f)(x)=\sup _{t>0}\left|B_{\delta, t}^{b}(f)(x)\right| .
$$

We also define

$$
B_{\delta, *}(f)(x)=\sup _{t>0}\left|B_{t}^{\delta}(f)(x)\right|,
$$

which is the maximal Bochner-Riesz operator (see [25]). Let $H$ be the space $H=\{h:\|h\|=$ $\left.\sup _{t>0}|h(t)|<\infty\right\}$, then

$$
B_{\delta, *}^{b}(f)(x)=\left\|B_{\delta, t}^{b}(f)(x)\right\|, \quad B_{*}^{\delta}(f)(x)=\left\|B_{t}^{\delta}(f)(x)\right\| .
$$

It is easy to see that $B_{\delta, *}^{b}$ satisfies the conditions of Theorems 1-6 (see [21]), thus Theorems 1-6 hold for $B_{\delta, * *}^{b}$. 


\section{References}

1. Garcia-Cuerva, J, Rubio de Francia, JL: Weighted Norm Inequalities and Related Topics. North-Holland Mathematics Studies, vol. 16. North-Holland, Amsterdam (1985)

2. Stein, EM: Harmonic Analysis: Real Variable Methods, Orthogonality and Oscillatory Integrals. Princeton University Press, Princeton (1993)

3. Torchinsky, A: Real Variable Methods in Harmonic Analysis. Pure and Applied Math., vol. 123. Academic Press, New York (1986)

4. Coifman, RR, Rochberg, R, Weiss, G: Factorization theorems for Hardy spaces in several variables. Ann. Math. 103, 611-635 (1976)

5. Pérez, C: Endpoint estimate for commutators of singular integral operators. J. Funct. Anal. 128, 163-185 (1995)

6. Pérez, C, Trujillo-Gonzalez, R: Sharp weighted estimates for multilinear commutators. J. Lond. Math. Soc. 65, 672-692 (2002)

7. Di Fazio, G, Ragusa, MA: Interior estimates in Morrey spaces for strong solutions to nondivergence form equations with discontinuous coefficients. J. Funct. Anal. 112, 241-256 (1993)

8. Chanillo, S: A note on commutators. Indiana Univ. Math. J. 31, 7-16 (1982)

9. Chen, WG: Besov estimates for a class of multilinear singular integrals. Acta Math. Sin. 16, 613-626 (2000)

10. Janson, S: Mean oscillation and commutators of singular integral operators. Ark. Math. 16, 263-270 (1978)

11. Paluszynski, M: Characterization of the Besov spaces via the commutator operator of Coifman, Rochberg and Weiss. Indiana Univ. Math. J. 44, 1-17 (1995)

12. Chang, DC, Li, JF, Xiao, J: Weighted scale estimates for Calderón-Zygmund type operators. Contemp. Math. 446, 61-70 (2007)

13. Lin, Y: Sharp maximal function estimates for Calderón-Zygmund type operators and commutators. Acta Math. Sci. Ser. A Chin. Ed. 31, 206-215 (2011)

14. Peetre, J. On convolution operators leaving $L^{p, \lambda}$-spaces invariant. Ann. Mat. Pura Appl. 72, 295-304 (1966)

15. Peetre, J: On the theory of $L^{p, \lambda}$-spaces. J. Funct. Anal. 4, 71-87 (1969)

16. Chiarenza, F, Frasca, M: Morrey spaces and Hardy-Littlewood maximal function. Rend. Mat. 7, 273-279 (1987)

17. Di Fazio, G, Ragusa, MA: Commutators and Morrey spaces. Boll. Unione Mat. Ital., A (7) 5, 323-332 (1991)

18. Liu, LZ: Interior estimates in Morrey spaces for solutions of elliptic equations and weighted boundedness for commutators of singular integral operators. Acta Math. Sci. 25(1), 89-94 (2005)

19. Mizuhara, T: Boundedness of some classical operators on generalized Morrey spaces. In: Harmonic Analysis. ICM-90 Satellite Conference Proceedings (Sendai, 1990), pp. 183-189 (1991)

20. Coifman, RR, Rochberg, R: Another characterization of BMO. Proc. Am. Math. Soc. 79, $249-254$ (1980)

21. Liu, LZ: The continuity of commutators on Triebel-Lizorkin spaces. Integral Equ. Oper. Theory 49, 65-76 (2004)

22. Liu, LZ: Triebel-Lizorkin space estimates for multilinear operators of sublinear operators. Proc. Indian Acad. Sci. Math. Sci. 113, 379-393 (2003)

23. Liu, LZ: Boundedness for multilinear Littlewood-Paley operators on Triebel-Lizorkin spaces. Methods Appl. Anal. 10(4), 603-614 (2004)

24. Torchinsky, A, Wang, S: A note on the Marcinkiewicz integral. Colloq. Math. 60(61), 235-243 (1990)

25. Lu, SZ: Four Lectures on Real HP Spaces. World Scientific, River Edge (1995)

10.1186/1029-242X-2014-172

Cite this article as: Zeng: Inequalities and boundedness for commutators related to integral operator with general kernel. Journal of Inequalities and Applications 2014, 2014:172

\section{Submit your manuscript to a SpringerOpen ${ }^{\circ}$ journal and benefit from:}

- Convenient online submission

- Rigorous peer review

- Immediate publication on acceptance

- Open access: articles freely available online

- High visibility within the field

- Retaining the copyright to your article 